\title{
Enhancing Situational Awareness by Ground Truthing with Historical Outbreaks
}

\author{
Lauren Castro*, Kirsten Taylor-McCabe, Eric Generous, Joseph Longo, Kristen \\ Margevicius, Reid Priedhorsky and Alina Deshpande
}

Los Alamos National Laboratory, Los Alamos, NM, USA

\section{Objective}

The goal of this project is to develop a cross platform app that contextualizes incoming information during an infectious disease outbreak based on historical data. The app makes use of a surveillance window concept in order to support decision making. This effort is part of a larger project with the goal of developing reference tools and analytics to provide decision-makers with timely information to predict, prepare for, and mitigate the spread of disease.

\section{Introduction}

Los Alamos National Laboratory (LANL) has been funded by the Defense Threat Reduction Agency to develop tools that enhance situational awareness in infectious disease surveillance. We have applied the concept of the surveillance window to the development of a cross platform app (SWAP). This app allows the user to place information on case counts or disease occurrence in a specific location within the context of a historical outbreak curve to help determine whether prevention or mitigation action should be taken. By placing a frame of reference for where a case count is during an outbreak (in the early, peak, or late stages) and indicating whether the unfolding events are still within a surveillance window that would allow for feasible control, the app provides enhanced situational awareness of a decision maker. This tool therefore increases the granularity of situational awareness available to any user in the global biosurveillance community.

\section{Methods}

We used a stepwise approach illustrated in Figure 1 for developing the app for cholera. Our first step was identifying historical case studies in diverse endemic and non-endemic locations, to generate a portfolio of historic outbreak curves. We conducted a literature review to contextualize each outbreak for economic, environmental, and political determinants that influenced the duration and spread of the epidemic. In addition we defined a surveillance window and identified available data streams that were available during and after the surveillance window. The second step was to conduct a cross-case study analysis of the outbreaks to identify specific input parameters that would allow the app to sort through the portfolio of historic outbreak curves and generate the best match based on the input information. Our third step was to design the application with end users in mind who have public health and epidemiological backgrounds. The fourth step was to implement the application functions that would match historical outbreaks with input queries, including calculations for percent match and identification of current placement. Each technical choice for the application was for free and open-source software that is heavily used world-wide.

\section{Results}

Results in this abstract are presented for the development of the SWAP prototype version for cholera. Our cross case-study analysis of 20 different cholera outbreaks demonstrated that a single representative curve and single surveillance window cannot be established for all cholera outbreaks. We evaluated 13 different input parameters as queries to sort between historical outbreaks, and ultimately selected four: case counts over time, duration since index case, regional location, and endemicity of the disease. Common factors in cholera epidemics, such as low levels of water infrastructure and malnutrition, while present in most outbreaks, do not help distinguish between outbreaks. Operational evaluation of the app for cholera will be the next step for refinement of the app.

\section{Conclusions}

Data obtained in isolation have little value to a decision maker. An important need of biosurveillance efforts is to have a frame of reference in order to determine infectious disease risk easier and faster. The developing SWAP provides a more robust understanding of rapidly unfolding events through historic contextualization and graphic visualization.

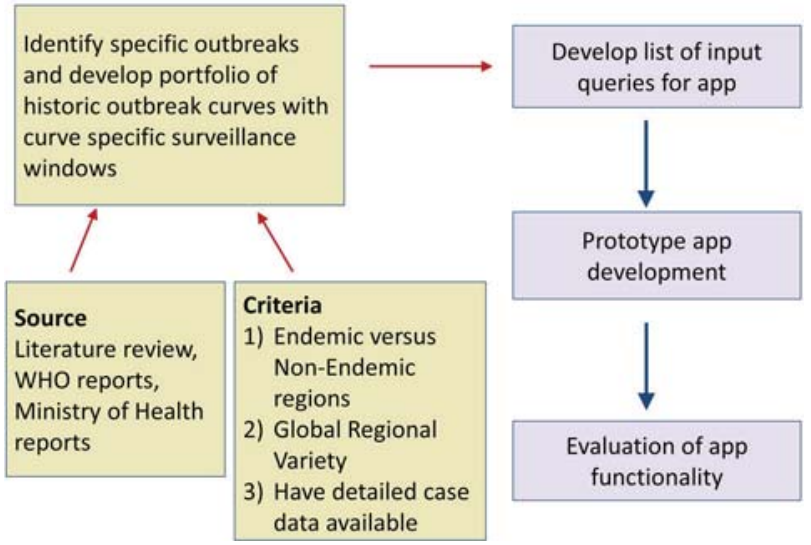

Figure 1: The overall approach to the development of the SWAP

\section{Keywords}

Surveillance Window; Biosurveillance; Historical Outbreaks; Situational Awareness

\section{Acknowledgments}

This project is supported by the Chemical and Biological Technologies Directorate Joint Science and Technology Office (JSTO), Defense Threat Reduction Agency (DTRA).

\section{*Lauren Castro \\ E-mail: Icastro@lanl.gov}

